\title{
RIO-TYPE INEQUALITY FOR THE EXPECTATION OF PRODUCTS OF RANDOM VARIABLES
}

\author{
B. L. S. PRAKASA RAO
}

Received 26 July 2004

We develop an inequality for the expectation of a product of $n$ random variables generalizing the recent work of Dedecker and Doukhan (2003) and the earlier results of Rio (1993).

\section{Introduction}

Let $(\Omega, \mathscr{F}, P)$ be a probability space and let $(X, Y)$ be a bivariate random vector defined on it. Suppose that $E\left(X^{2}\right)<\infty$ and $E\left(Y^{2}\right)<\infty$. Hoeffding proved that

$$
\operatorname{Cov}(X, Y)=\int_{\mathbb{R}^{2}}[P(X \leq x, Y \leq y)-P(X \leq x) P(Y \leq y)] d x d y .
$$

In [5], Lehmann gave a simple proof of this identity and used it in his study of some concepts of dependence. This identity was generalized to functions $h(X)$ and $g(Y)$ with $E\left[h^{2}(X)\right]<\infty$ and $E\left[g^{2}(Y)\right]<\infty$ and with finite derivatives $h^{\prime}(\cdot)$ and $g^{\prime}(\cdot)$ by Newman [6]. Multidimensional versions of these results were proved by Block and Fang [1], Yu [13], and more recently by Prakasa Rao [7]. Related covariance identities for exponential and other distributions are given by Prakasa Rao in $[9,10]$.

Suppose that $\mathcal{M}$ is a sub- $\sigma$-algebra of $\mathscr{F}$ and $Y$ is measurable with respect to $\mathcal{M}$. Let $\sigma(X)$ be the sub- $\sigma$-algebra generated by the random variable $X$. Define

$$
\alpha(\mathcal{M}, X)=\sup \{|P(A \cap B)-P(A) P(B)|, A \in \mathcal{M}, B \in \sigma(X)\} .
$$

Define

$$
\begin{gathered}
Q_{X}(u)=\inf \{x: P(|X|>x) \leq u\}, \\
G_{X}(s)=\inf \left\{z: \int_{0}^{z} Q_{X}(t) d t \geq s\right\}, \\
H_{X, Y}(s)=\inf \left\{t: E\left(|X| I_{[|Y|>t]}\right) \leq s\right\} .
\end{gathered}
$$


Rio [11] proved that

$$
|\operatorname{Cov}(X, Y)| \leq 2 \int_{0}^{\alpha(\mu, X) / 2} Q_{Y}(u) Q_{X}(u) d u .
$$

Related results are given in [12, page 9]. These results were generalized by Bradley [2] for a strong-mixing process and by Prakasa Rao [8] for $r$ th-order joint cumulant under $r$ th-order strong mixing. In a recent work, Dedecker and Doukhan [3] proved that

$$
|E(X Y)| \leq \int_{0}^{\|E(X \mid \mathcal{M})\|_{1}} H_{X, Y}(t) d t \leq \int_{0}^{\|E(X \mid \mathcal{M})\|_{1}} Q_{Y} o G_{X}(t) d t
$$

and obtained an improved version of the above inequality. If $X_{i}, 1 \leq i \leq n$, are positivevalued random variables, it is easy to see that

$$
E\left(X_{1} X_{2} \cdots X_{n}\right) \leq \int_{0}^{1} Q_{X_{1}}(u) Q_{X_{2}}(u) \cdots Q_{X_{n}}(u) d u
$$

For a proof, see [12, Lemma 2.1, page 35].

We now obtain an improved version of the above inequality following the techniques of Dedecker and Doukhan [3] and Block and Fang [1].

\section{Main result}

Let $\left\{X_{i}, 1 \leq i \leq n\right\}$ be a sequence of nonnegative random variables defined on a probability space $(\Omega, \mathscr{F}, P)$. Then the random variable $X_{i}$ can be represented in the form

$$
X_{i}=\int_{0}^{\infty} I_{\left(x_{i}, \infty\right)}\left(X_{i}\right) d x_{i}
$$

where

$$
I_{\left(x_{i}, \infty\right)}\left(X_{i}\right)= \begin{cases}1 & \text { if } X_{i}>x_{i} \\ 0 & \text { if } X_{i} \leq x_{i}\end{cases}
$$

Hence

$$
\begin{aligned}
E\left(X_{1} X_{2} \cdots X_{n}\right) & =E\left[X_{1} \Pi_{i=2}^{n} \int_{0}^{\infty} I_{\left(x_{i}, \infty\right)}\left(X_{i}\right) d x_{i}\right] \\
& =\int_{\mathbb{R}_{+}^{n-1}} E\left[X_{1} \Pi_{i=2}^{n} I_{\left(x_{i}, \infty\right)}\left(X_{i}\right)\right] d x_{2} \cdots d x_{n} \\
& =\int_{\mathbb{R}_{+}^{n-1}} E\left[X_{1} I_{\left[X_{i}>x_{i}, 2 \leq i \leq n\right]}\left(X_{2}, \ldots, X_{n}\right)\right] d x_{2} \cdots d x_{n}
\end{aligned}
$$

by the Fubini's theorem, where $\mathbb{R}_{+}^{n-1}=\left\{\left(x_{2}, \ldots, x_{n}\right): x_{i} \geq 0,2 \leq i \leq n\right\}$. Observe that

$$
E\left(X_{1} I_{\left[X_{i}>x_{i}, 2 \leq i \leq n\right]}\left(X_{2}, \ldots, X_{n}\right)\right) \leq \min \left(E\left[X_{1}\right], E\left(X_{1} I_{\left[X_{i}>x_{i}, 2 \leq i \leq n\right]}\left(X_{2}, \ldots, X_{n}\right)\right)\right)
$$


and hence

$$
E\left(X_{1} X_{2} \cdots X_{n}\right) \leq \int_{\mathbb{R}_{+}^{n-1}}\left\{\int_{0}^{E X_{1}} \chi_{\left(E\left[X_{1} I_{\left[X_{i}>x_{i}, 2 \leq i \leq n\right]}\left(X_{2}, \ldots, X_{n}\right)\right]>u\right)}(u) d u\right\} d x_{2} \cdots d x_{n} .
$$

Here $\chi_{A}(\cdot)$ denotes the indicator function of the set $A$. Let

$$
g_{X_{1}}\left(x_{2}, \ldots, x_{n}\right)=E\left[X_{1} I_{\left[X_{i}>x_{i}, 2 \leq i \leq n\right]}\left(X_{2}, \ldots, X_{n}\right)\right] .
$$

Then

$$
\begin{aligned}
E\left(X_{1} X_{2} \cdots X_{n}\right) & \leq \int_{\mathbb{R}_{+}^{n-1}}\left\{\int_{0}^{E X_{1}} \chi_{\left[g_{X_{1}}\left(x_{2}, \ldots, x_{n}\right)>u\right]}(u) d u\right\} d x_{2} \cdots d x_{n} \\
& =\int_{0}^{E\left(X_{1}\right)}\left\{\int_{\left[\left(x_{2}, \ldots, x_{n}\right): g_{X_{1}}\left(x_{2}, \ldots, x_{n}\right)>u\right]} 1 d x_{2} \cdots d x_{n}\right\} d u .
\end{aligned}
$$

Let

$$
H_{X_{1}, X_{2}, \ldots, X_{n}}(u)=\lambda\left[\left(x_{2}, \ldots, x_{n}\right): g_{X_{1}}\left(x_{2}, \ldots, x_{n}\right)>u\right]
$$

where $\lambda$ is the Lebesgue measure on the space $\mathbb{R}_{+}^{n-1}$. Hence

$$
E\left(X_{1} X_{2} \cdots X_{n}\right) \leq \int_{0}^{E\left(X_{1}\right)} H_{X_{1}, X_{2}, \ldots, X_{n}}(u) d u
$$

Observe that

$$
g_{X_{1}}\left(x_{2}, \ldots, x_{n}\right)=E\left[X_{1} I_{\left[X_{i}>x_{i}, 2 \leq i \leq n\right]}\left(X_{2}, \ldots, X_{n}\right)\right] \leq \int_{0}^{E\left[I_{\left[X_{i}>x_{i}, 2 \leq i \leq n\right]}\left(X_{2}, \ldots, X_{n}\right)\right]} Q_{X_{1}}(u) d u
$$

from the Fréchet's inequality [4]. Here $Q_{X_{1}}(\cdot)$ is the generalized inverse of the function $T_{X_{1}}(x)=P\left(X_{1}>x\right)$ as defined earlier. Let

$$
M_{X_{1}}(y)=\int_{0}^{y} Q_{X_{1}}(t) d t
$$

Observe that $M_{X_{1}}(\cdot)$ is nondecreasing in $y$. Let $G_{X_{1}}(u)=\inf \left\{z: M_{X_{1}}(z) \geq u\right\}$ as defined earlier. Let

$$
T_{X_{2}, \ldots, X_{n}}\left(x_{2}, \ldots, x_{n}\right)=P\left(X_{i}>x_{i}, 2 \leq i \leq n\right) .
$$

Note that

$$
\begin{aligned}
g_{X_{1}}\left(x_{2}, \ldots, x_{n}\right) & \leq M_{X_{1}}\left(E\left(I_{\left[X_{i}>x_{i}, 2 \leq i \leq n\right]}\left(X_{2}, \ldots, X_{n}\right)\right)\right), \\
g_{X_{1}}\left(x_{2}, \ldots, x_{n}\right)>u & \Longrightarrow M_{X_{1}}\left(E\left(I_{\left[X_{i}>x_{i}, 2 \leq i \leq n\right]}\left(X_{2}, \ldots, X_{n}\right)\right)\right)>u \\
& \Longrightarrow E\left(I_{\left[X_{i}>x_{i}, 2 \leq i \leq n\right]}\left(X_{2}, \ldots, X_{n}\right)\right)>G_{X_{1}}(u) \\
& \Longrightarrow P\left[X_{i}>x_{i}, 2 \leq i \leq n\right]>G_{X_{1}}(u) .
\end{aligned}
$$


Hence the set

$$
\left[\left(x_{2}, \ldots, x_{n}\right) \in \mathbb{R}_{+}^{n-1}: g_{X_{1}}\left(x_{2}, \ldots, x_{n}\right)>u\right]
$$

is contained in the set

$$
\left[\left(x_{2}, \ldots, x_{n}\right) \in \mathbb{R}_{+}^{n-1}: P\left(X_{i}>x_{i}, 2 \leq i \leq n\right)>G_{X_{1}}(u)\right]
$$

In particular, it follows that the Lebesgue measure of the former set is less than or equal to that of the latter. Let

$$
Q_{X_{2}, \ldots, X_{n}}^{*}\left(G_{X_{1}}(u)\right)
$$

denote the Lebesgue measure of the set (2.15).

Then

$$
H_{X_{1}, X_{2}, \ldots, X_{n}}(u) \leq Q_{X_{2}, \ldots, X_{n}}^{*}\left(G_{X_{1}}(u)\right)
$$

for all $0 \leq u \leq 1$. Hence

$$
E\left(X_{1} X_{2} \cdots X_{n}\right) \leq \int_{0}^{E\left(X_{1}\right)} Q_{X_{2}, \ldots, X_{n}}^{*}\left(G_{X_{1}}(u)\right) d u .
$$

We have proved the following inequality.

Theorem 2.1. Let $X_{i}, 1 \leq i \leq n$, be nonnegative random variables defined on a probability space $(\Omega, \mathscr{F}, P)$. Then

$$
E\left(X_{1} X_{2} \cdots X_{n}\right) \leq \int_{0}^{E\left(X_{1}\right)} H_{X_{1}, X_{2}, \ldots, X_{n}}(u) d u \leq \int_{0}^{E\left(X_{1}\right)} Q_{X_{2}, \ldots, X_{n}}^{*} o G_{X_{1}}(u) d u,
$$

where the functions $H, Q^{*}$, and $G$ are as defined earlier.

\section{Applications}

We now suppose that the random variables $\left\{X_{i}, 1 \leq i \leq n\right\}$ are arbitrary but with

$$
E\left|X_{1} X_{2} \cdots X_{n}\right|<\infty
$$

Define

$$
\begin{gathered}
g_{X_{1}}\left(x_{2}, \ldots, x_{n}\right)=E\left(\left|X_{1}\right| I_{\left[\left|X_{i}\right|>x_{i}, 2 \leq i \leq n\right]}\left(X_{2}, \ldots, X_{n}\right)\right), \\
H_{X_{1}, X_{2}, \ldots, X_{n}}(u)=\lambda\left[\left(x_{2}, \ldots, x_{n}\right): g_{X_{1}}\left(x_{2}, \ldots, x_{n}\right) \leq u\right], \\
T_{X_{2}, \ldots, X_{n}}\left(x_{2}, \ldots, x_{n}\right)=P\left(\left|X_{i}\right|>x_{i}, 2 \leq i \leq n\right),
\end{gathered}
$$

and define $M_{X_{1}}(\cdot), Q_{X_{1}}(\cdot), Q_{X_{2}, \ldots, X_{n}}^{*}$, and $G_{X_{1}}$ accordingly. The following theorem follows by arguments analogous to those given in Section 2 . 
Theorem 3.1. Let $X_{i}, 1 \leq i \leq n$, be arbitrary random variables defined on a probability space $(\Omega, \mathscr{F}, P)$. Then

$$
E\left(\left|X_{1} X_{2} \cdots X_{n}\right|\right) \leq \int_{0}^{E\left(\left|X_{1}\right|\right)} H_{X_{1}, X_{2}, \ldots, X_{n}}(u) d u \leq \int_{0}^{E\left(\left|X_{1}\right|\right)} Q_{X_{2}, \ldots, X_{n}}^{*} o G_{X_{1}}(u) d u,
$$

where the functions $H, Q^{*}$, and $G$ are as defined above.

In particular, for $n=2$, we have

$$
E\left(\left|X_{1} X_{2}\right|\right) \leq \int_{0}^{E\left(\left|X_{1}\right|\right)} H_{X_{1}, X_{2}}(u) d u \leq \int_{0}^{E\left(\left|X_{1}\right|\right)} Q_{X_{2}} o G_{X_{1}}(u) d u
$$

since $Q_{X}^{*}=Q_{X}$ for any univariate random variable $X$. Furthermore,

$$
G_{X_{1}-E\left(X_{1}\right)}(u) \geq G_{X_{1}}\left(\frac{u}{2}\right), \quad 0 \leq u \leq 1
$$

(cf. [3]). Hence

$$
E\left[\left|X_{1} X_{2}\right|\right] \leq \int_{0}^{G_{X_{1}}^{-1}\left(E\left(\left|X_{1}\right|\right) / 2\right)} Q_{X_{2}}(u) Q_{X_{1}}(u) d u .
$$

Therefore, for any two functions $f_{i}(\cdot), i=1,2$, with $f_{i}(0)=0$ such that $E\left|f_{1}\left(X_{1}\right) f_{2}\left(X_{2}\right)\right|<$ $\infty$, we obtain that

$$
E\left[\left|f_{1}\left(X_{1}\right) f_{2}\left(X_{2}\right)\right|\right] \leq \int_{0}^{G_{f_{1}\left(X_{1}\right)}^{-1}\left(E\left(\left|f_{1}\left(X_{1}\right)\right|\right) / 2\right)} Q_{f_{2}\left(X_{2}\right)}(u) Q_{f_{1}\left(X_{1}\right)}(u) d u .
$$

Applying Theorem 3.1 for the random variables $X_{1}-E\left(X_{1}\right), X_{2}, \ldots, X_{n}$, we get that

$$
E\left[\left|\left(X_{1}-E\left(X_{1}\right)\right) X_{2} \cdots X_{n}\right|\right] \leq \int_{0}^{E\left(\left|X_{1}-E\left(X_{1}\right)\right|\right)} Q_{X_{2}, \ldots, X_{n}}^{*} o G_{X_{1}-E\left(X_{1}\right)}(u) d u .
$$

But

$$
G_{X_{1}-E\left(X_{1}\right)}(u) \geq G_{X_{1}}\left(\frac{u}{2}\right), \quad u \geq 0
$$

(cf. [3]). Hence

$$
E\left[\left|\left(X_{1}-E\left(X_{1}\right)\right) X_{2} \cdots X_{n}\right|\right] \leq \int_{0}^{E\left(\left|X_{1}-E\left(X_{1}\right)\right|\right) / 2} Q_{X_{2}, \ldots, X_{n}}^{*} o G_{X_{1}}(u) d u .
$$

Observing that $G_{X_{1}}(\cdot)$ is the inverse of the function $M_{X_{1}}(y)=\int_{0}^{y} Q_{X_{1}}(t) d t$, it follows that

$$
E\left[\left|\left(X_{1}-E\left(X_{1}\right)\right) X_{2} \cdots X_{n}\right|\right] \leq \int_{0}^{G_{X_{1}}^{-1}\left(E\left(\left|X_{1}-E\left(X_{1}\right)\right|\right) / 2\right)} Q_{X_{2}, \ldots, X_{n}}^{*}(u) Q_{X_{1}}(u) d u
$$

Hence we have the following result. 
Theorem 3.2. Let $X_{i}, 1 \leq i \leq n$, be arbitrary random variables defined on a probability space $(\Omega, \mathscr{F}, P)$ with $E\left|X_{1}\right|<\infty$ and $E\left|X_{1} X_{2} \cdots X_{n}\right|<\infty$. Then (3.11) holds.

Observe that $Q_{X}^{*}=Q_{X}$ for any univariate random variable $X$. Let $n=2$ in Theorem 3.2. Then $Q_{X_{2}}^{*}=Q_{X_{2}}$ and the above result reduces to

$$
E\left[\left|\left(X_{1}-E\left(X_{1}\right)\right) X_{2}\right|\right] \leq \int_{0}^{G_{X_{1}}^{-1}\left(E\left(\left|X_{1}-E\left(X_{1}\right)\right|\right) / 2\right)} Q_{X_{2}}(u) Q_{X_{1}}(u) d u .
$$

As a further consequence, we get that

$$
E\left[\left|\left(X_{1}-E\left(X_{1}\right)\right)\left(X_{2}-E\left(X_{2}\right)\right)\right|\right] \leq \int_{0}^{G_{X_{1}}^{-1}\left(E\left(\left|X_{1}-E\left(X_{1}\right)\right|\right) / 2\right)} Q_{X_{2}-E\left(X_{2}\right)}(u) Q_{X_{1}}(u) d u .
$$

Since

$$
Q_{X_{2}-E\left(X_{2}\right)} \leq Q_{X_{2}}+E\left|X_{2}\right|
$$

we obtain that

$$
\begin{aligned}
& E\left[\left|\left(X_{1}-E\left(X_{1}\right)\right)\left(X_{2}-E\left(X_{2}\right)\right)\right|\right] \\
& \quad \leq \int_{0}^{G_{X_{1}}^{-1}\left(E\left(\left|X_{1}-E\left(X_{1}\right)\right|\right) / 2\right)} Q_{X_{2}}(u) Q_{X_{1}}(u) d u+E\left|X_{2}\right| \int_{0}^{G_{X_{1}}^{-1}\left(E\left(\left|X_{1}-E\left(X_{1}\right)\right|\right) / 2\right)} Q_{X_{1}}(u) d u .
\end{aligned}
$$

Let

$$
\alpha\left(X_{1}, X_{2}\right)=\max \left\{G_{X_{1}}^{-1}\left(\frac{E\left(\left|X_{1}-E\left(X_{1}\right)\right|\right)}{2}\right), G_{X_{2}}^{-1}\left(\frac{E\left(\left|X_{2}-E\left(X_{2}\right)\right|\right)}{2}\right)\right\} .
$$

Then it follows that

$$
\begin{aligned}
& E\left[\left|\left(X_{1}-E\left(X_{1}\right)\right)\left(X_{2}-E\left(X_{2}\right)\right)\right|\right] \\
& \leq \int_{0}^{\alpha\left(X_{1}, X_{2}\right)} Q_{X_{1}}(u) Q_{X_{2}}(u) d u+\frac{1}{2}\left(E\left|X_{1}\right| \int_{0}^{\alpha\left(X_{1}, X_{2}\right)} Q_{X_{1}}(u) d u+E\left|X_{2}\right| \int_{0}^{\alpha\left(X_{1}, X_{2}\right)} Q_{X_{2}}(u) d u\right) .
\end{aligned}
$$

This inequality is different from the inequality in [12, page 9].

Let $f_{1}$ and $f_{2}$ be differentiable functions on $\mathbb{R}_{+}$with $f_{i}(0)=0$. Let $X_{i}, i=1,2$, be nonnegative random variables. Suppose that $E\left[f_{i}^{2}\left(X_{i}\right)\right]<\infty, i=1,2$. It is easy to see that

$$
f_{i}\left(X_{i}\right)=\int_{0}^{\infty} f_{i}^{\prime}\left(X_{i}\right) I_{\left(x_{i}, \infty\right)}\left(X_{i}\right) d x_{i}
$$

Then

$$
\begin{aligned}
E\left(f_{1}\left(X_{1}\right) f_{2}\left(X_{2}\right)\right) & =E\left[f_{1}\left(X_{1}\right) \int_{0}^{\infty} f_{2}^{\prime}\left(X_{2}\right) I_{\left(x_{2}, \infty\right)}\left(X_{2}\right) d x_{2}\right] \\
& =\int_{\mathbb{R}_{+}} E\left[f_{1}\left(X_{1}\right) f_{2}^{\prime}\left(X_{2}\right) I_{\left(x_{2}, \infty\right)}\left(X_{2}\right)\right] d x_{2}
\end{aligned}
$$


by the Fubini's theorem. Observe that

$$
\begin{aligned}
& E\left(\left|f_{1}\left(X_{1}\right) f_{2}^{\prime}\left(X_{2}\right)\right| I_{\left[X_{2}>x_{2}\right]}\left(X_{2}\right)\right) \\
& \quad \leq \min \left(E\left[\left|f_{1}\left(X_{1}\right) f_{2}^{\prime}\left(X_{2}\right)\right|\right], E\left(\left|f_{1}\left(X_{1}\right) f_{2}^{\prime}\left(X_{2}\right)\right| I_{\left[X_{2}>x_{2}\right]}\left(X_{2}\right)\right)\right)
\end{aligned}
$$

and hence

$$
\begin{aligned}
& \left|E\left(f_{1}\left(X_{1}\right) f_{2}\left(X_{2}\right)\right)\right| \\
& \quad \leq \int_{\mathbb{R}+}\left\{\int_{0}^{E\left[\left|f_{1}\left(X_{1}\right) f_{2}^{\prime}\left(X_{2}\right)\right|\right]} \chi_{\left(E\left[\left|f_{1}\left(X_{1}\right) f_{2}^{\prime}\left(X_{2}\right)\right| I_{\left[X_{2}>x_{2}\right]}\left(X_{2}\right)\right]>u\right)}(u) d u\right\} d x_{2} .
\end{aligned}
$$

Here $\chi_{A}(\cdot)$ denotes the indicator function of the set $A$. Let

$$
g_{f_{1}\left(X_{1}\right), f_{2}^{\prime}\left(X_{2}\right)}\left(x_{2}\right)=E\left[\left|f_{1}\left(X_{1}\right) f_{2}^{\prime}\left(X_{2}\right)\right| I_{\left[X_{2}>x_{2}\right]},\left(X_{2}\right)\right] .
$$

Then

$$
\begin{aligned}
\left|E\left(f_{1}\left(X_{1}\right) f_{2}\left(X_{2}\right)\right)\right| & \leq \int_{\mathbb{R}_{+}}\left\{\int_{0}^{E\left[\left|f_{1}\left(X_{1}\right) f_{2}^{\prime}\left(X_{2}\right)\right|\right]} \chi_{\left(\left[g_{f_{1}\left(X_{1}\right), f_{2}^{\prime}\left(X_{2}\right)}\left(x_{2}\right)\right]>u\right)}(u) d u\right\} d x_{2} \\
& \leq \int_{0}^{E\left[\left|f_{1}\left(X_{1}\right) f_{2}^{\prime}\left(X_{2}\right)\right|\right]}\left\{\int_{\left[x_{2}: g_{f_{1}\left(X_{1}\right), f_{2}^{\prime}\left(X_{2}\right)}\left(x_{2}\right)>u\right]} 1 d x_{2}\right\} d u .
\end{aligned}
$$

Let

$$
H_{f_{1}\left(X_{1}\right), f_{2}^{\prime}\left(X_{2}\right)}(u)=\inf \left\{x_{2}: g_{f_{1}\left(X_{1}\right), f_{2}^{\prime}\left(X_{2}\right)}\left(x_{2}\right) \leq u\right\}
$$

Then it follows that

$$
\left|E\left(f_{1}\left(X_{1}\right) f_{2}\left(X_{2}\right)\right)\right| \leq \int_{0}^{E\left[\left|f_{1}\left(X_{1}\right) f_{2}^{\prime}\left(X_{2}\right)\right|\right]} H_{f_{1}\left(X_{1}\right), f_{2}^{\prime}\left(X_{2}\right)}(u) d u .
$$

An analogous inequality holds by interchanging $f_{1}\left(X_{1}\right)$ and $f_{2}\left(X_{2}\right)$ :

$$
\left|E\left(f_{1}\left(X_{1}\right) f_{2}\left(X_{2}\right)\right)\right| \leq \int_{0}^{E\left[\left|f_{1}^{\prime}\left(X_{1}\right) f_{2}\left(X_{2}\right)\right|\right]} H_{f_{1}^{\prime}\left(X_{1}\right), f_{2}\left(X_{2}\right)}(u) d u
$$

\section{References}

[1] H. W. Block and Z. B. Fang, A multivariate extension of Hoeffding's lemma, Ann. Probab. 16 (1988), no. 4, 1803-1820.

[2] R. C. Bradley, A covariance inequality under a two-part dependence assumption, Statist. Probab. Lett. 30 (1996), no. 4, 287-293.

[3] J. Dedecker and P. Doukhan, A new covariance inequality and applications, Stochastic Process. Appl. 106 (2003), no. 1, 63-80.

[4] M. Fréchet, Sur la distance de deux lois de probabilité, C. R. Acad. Sci. Paris 244 (1957), 689-692 (French).

[5] E. L. Lehmann, Some concepts of dependence, Ann. Math. Statist. 37 (1966), 1137-1153.

[6] C. M. Newman, Normal fluctuations and the FKG inequalities, Comm. Math. Phys. 74 (1980), no. 2, 119-128. 


\section{Rio-type inequality}

[7] B. L. S. Prakasa Rao, Hoeffding identity, multivariance and multicorrelation, Statistics 32 (1998), no. 1, 13-29.

[8] B Bounds for r th order joint cumulant under $r$ th order strong mixing, Statist. Probab. Lett. 43 (1999), no. 4, 427-431.

[9] Covariance identities for exponential and related distributions, Statist. Probab. Lett. 42 (1999), no. 3, 305-311.

[10] Some covariance identities, inequalities and their applications: a review, Proc. Indian Nat. Sci. Acad. Part A 66 (2000), no. 5, 537-543.

[11] E. Rio, Covariance inequalities for strongly mixing processes, Ann. Inst. H. Poincaré Probab. Statist. 29 (1993), no. 4, 587-597.

[12] Théorie Asymptotique des Processus Aléatoires Faiblement Dépendants, Springer, Paris, 2000.

[13] H. Yu, A Glivenko-Cantelli lemma and weak convergence for empirical processes of associated sequences, Probab. Theory Related Fields 95 (1993), no. 3, 357-370.

B. L. S. Prakasa Rao: Theoretical Statistics and Mathematics Unit, Indian Statistical Institute, New Delhi 110 016, India

E-mail address: blsp@isid.ac.in 